\title{
Combinatorics 2010
}

The Italian Combinatorics conferences began in Rome in 1981, organised by Giuseppe Tallini, continued in 1982 at Passo della Mendola and have happened every 2 years since then.

The 16th in this sequence, Combinatorics 2010, was organised by E. Dedò, E. Munarini, C. Perelli-Cippo and N. Zagaglia from the Mathematics Department of the Politecnico di Milano. The conference was dedicated to Adriano Barlotti and took place from 27th June to 3rd July 2010 in Verbania on the shores of Lago Maggiore. Of the 18 invited talks, this volume presents articles based on 14 of them.

These articles cover many aspects of Combinatorics:

Coding theory: T. Maruta

Colouring problems: C. Bujtás and Z. Tuza

Design theory: (a) C.J. Colbourn; (b) D. Jungnickel

Extremal set theory: A. Blokhuis, A.E. Brouwer, T. Szőnyi and Z. Weiner

Exterior algebras: A. Brini and F. Regonati

Frameworks: B. Jackson

Generalised quadrangles: K. Thas

Polar spaces: (a) B. De Bruyn; (b) K. Metsch

Projective planes: (a) G. Lunardon; (b) K. Strambach and A. Barlotti ${ }^{\dagger}$

Riordan arrays: R. Sprugnoli

Veronese and Segre varieties: J.A. Thas and H. Van Maldeghem

J. W. P. Hirschfeld

JWPH@sussex.ac.uk 